\title{
Pollution des eaux superficielles et des nappes en milieu urbain : cas de la zone industrielle de Douala-Bassa (Cameroun)
}

\author{
L. KENGNI ${ }^{1 *}$, P. TEMATIO ${ }^{1}$, K. FILALI RHARRASSI ${ }^{2}$, J. TEPOULE NGUEKE ${ }^{3}$, \\ E. I. TSAFACK ${ }^{1}$, T. L. MBOUMI ${ }^{1}$ et S. MOUNIER ${ }^{2}$ \\ ${ }^{I}$ Université de Dschang, Laboratoire de géologie appliquée, LABOGA, BP 67 Dschang. \\ ${ }^{2}$ Université du Sud Toulon Var, Laboratoire PROTEE, BP 20132 - 83957 LA GARDE Cedex - France. \\ ${ }^{3}$ Université de Dschang, CEREHT, BP 222 Dschang. \\ *Auteur correspondant ; E-mail: lkengni@yahoo.fr, Tél 0023799819122.
}

\section{RESUME}

Une étude a été menée dans la zone industrielle de Douala-Bassa afin d'évaluer l'ampleur de la pollution des cours d'eau et des nappes superficielles et de prédire la vulnérabilité de ces dernières. L'étude est basée sur l'analyse des eaux issues de deux campagnes de terrain portant chacune sur 7 prélèvements dans les eaux de surface et 8 dans les eaux souterraines. Outre les analyses physico-chimiques de base, la particularité de l'étude a porté sur la caractérisation de la matière organique et la dynamique de métaux lourds, notamment le plomb, le nickel et le cuivre. Les résultats montrent que les eaux de surface sont neutres ( $\mathrm{pH}$ 6,2), peu minéralisées (conductivité moyenne $262 \mu \mathrm{S} / \mathrm{cm}$ ) et peu turbides (7 NTU) à l'amont. Elles s'enrichissent ensuite dans le réseau où elles deviennent acides ( $\mathrm{pH} 5,8)$, plus minéralisées (623 à $818 \mu \mathrm{S} / \mathrm{cm}$ ) suite aux apports générés par les activités industrielles et domestiques. Les eaux de surface sont polluées (carbonique organique total, 13,0 $\mathrm{mg} / \mathrm{l}$; turbidité $168 \mathrm{NTU}$ ). Une source potentielle de pollution au nickel et en matière organique est observée dans les eaux souterraines qui compromet l'exploitation durable de cette ressource en eau.

(C) 2012 International Formulae Group. All rights reserved.

Mots clés: Source, puits, métaux lourds, carbone organique, pollution.

\section{INTRODUCTION}

La pollution de l'eau dans nos villes est préoccupante, d'autant plus que l'eau est le milieu récepteur final de toutes les formes de pollution notamment de l'air et du sol. Les eaux superficielles ainsi que des aquifères font très souvent les frais des pollutions diverses dont les causes sont nombreuses et variées, les plus connues étant celles liées à l'activité humaine et notamment l'urbanisation et l'industrialisation. L'urbanisation, de par la forte consommation de l'eau qu'elle engendre, entraîne la production d'une quantité considérable d'eaux usées domestiques dont la gestion est aléatoire en l'absence de système de collecte et de traitement des eaux usées (égouts collecteurs, stations d'épuration). Les eaux des aquifères sont en effet utilisées pour les tâches ménagères: nettoyage, arrosage, baignade, lessive et cuisson. Après leur utilisation, ces eaux sont rejetées directement dans le cours d'eau, le système de drainage étant unitaire. A cela il faut ajouter l'industrialisation qui se développe au gré 
d'investissements capitalistes peu soucieux de la protection de l'environnement (Champaud, 1983). Et ceci est d'autant plus flagrant que les industries qui s'installent ne prennent très souvent aucune disposition pour traiter leurs effluents liquides ou gazeux avant les rejets dans le milieu naturel. Plus grave encore, les zones industrielles ne se démarquent généralement pas des zones d'habitation, ce qui amplifie même les effets des pollutions sur les populations riveraines. Selon l'ONUDI (Organisation des Nations Unies pour le Développement Industriel), la majorité des unités industrielles camerounaises ne sont pas équipées des systèmes de prétraitement de leurs déchets industriels. Par conséquent, elles contribuent, en association avec l'urbanisation, à la charge hydraulique des rejets et véhiculent d'importantes quantités de substances toxiques susceptibles de contaminer les eaux superficielles et profondes (Serghini et al., 2003). La ville de Douala, avec une population de plus de 2,5 millions d'habitants, n'échappe pas à ce constat. Moins de la moitié de sa population a accès à l'eau potable fournie par la CAMWATER (INS, 2005). De ce fait, une grande partie de la population a recours aux méthodes alternatives d'approvisionnement en eau, sans pour autant que leur qualité soit réellement prise en compte (Djeuda Tchapnga et al., 1991; Koul'a, 2006 ; Baok, 2007 ; Ndjama et al., 2008 ; Temgoua et al., 2009). Les eaux de surface de l'agglomération de Douala sont de plus en plus polluées (Assonfack, 2002 ; Ketchemen-Tandia et al., 2004 ; Djuikom et al., 2009). En plus, le relief plat de la ville, caractéristique des basses terres du littoral camerounais, entraîne un mauvais drainage. Ainsi, l'écoulement des eaux usées n'y est pas favorisé, ce qui entraîne leur stagnation, leur infiltration dans le sol et une pollution progressive des nappes d'eau souterraines qui dans ce cas précis sont proches de la surface. La matière organique traversant le système eau-sol et surtout les métaux lourds sont des parfaits témoins de la pollution durable de l'eau, en raison de leur stabilité et de leur faculté à s'accumuler dans la chaîne alimentaire (Forstner et Wittmann, 1981). Au-delà d'un seuil, ils peuvent altérer l'organisation et la structure des organismes aquatiques dans l'écosystème. Les effets à long terme de ces derniers paramètres soulignent également l'un des aspects les plus inquiétants pour la santé environnementale dans les quartiers à habitat spontané (Jourdan et al., 2005 ; Baque, 2006).

C'est dans le but d'évaluer l'ampleur de la pollution tant sur les cours d'eau que sur les eaux souterraines et de prédire la vulnérabilité de la nappe par rapport aux apports de surface que cette étude est entreprise sur le bassin versant élémentaire de la Mgoua situé en plein cœur de la zone industrielle de Douala-Bassa. Cette zone est en effet marquée par une intense activité industrielle et une quasiabsence des systèmes d'évacuations des rejets. L'étude a pour objectif principal d'identifier les sources potentielles de pollution et de rechercher la relation entre les eaux de surface et des nappes. L'ampleur de la pollution peut s'apprécier au regard des indicateurs de la qualité physico-chimique de l'eau (la couleur, la température, le $\mathrm{pH}$, la minéralisation/ conductivité). L'originalité de l'étude porte sur la caractérisation de la matière organique traversant le système eau-sol et la dynamique des métaux lourds, notamment le plomb, le nickel et le cuivre.

\section{MATERIEL ET METHODES \\ Le site d'étude}

La zone d'étude se situe dans les basses terres du littoral camerounais (Figure 1). Le bassin versant de la Mgoua d'allongement principal NE-SW s'étend sur une superficie de 780 ha incluant les quartiers Nylon, Madagascar, Brazaville, CCC, UCB, SOCARTO. Les coordonnées à l'exutoire sont $4^{\circ} 01^{\prime} 40.64^{\prime \prime} \mathrm{N}$ et $9^{\circ} 44^{\prime} 10.13^{\prime \prime} \mathrm{E}$. Les altitudes ne dépassent pas $100 \mathrm{~m}$. Le climat est équatorial, de type camerounien, sous type côtier, caractérisé par des précipitations moyennes annuelles de l'ordre de $4000 \mathrm{~mm}$. La température moyenne annuelle est de 
l'ordre de $27,0{ }^{\circ} \mathrm{C}$. Elle varie entre $25,1{ }^{\circ} \mathrm{C}$ en Août et $28,4{ }^{\circ} \mathrm{C}$ en février (Figure 2). Les vents y sont rarement violents, la vitesse moyenne est de l'ordre de $1 \mathrm{~m} / \mathrm{s}$ tandis que la vitesse maximale ne dépasse pas $14 \mathrm{~m} / \mathrm{s}$. Sur le plan géologique, les séries sédimentaires vont de l'Albo-Aptien au Quaternaire (Njike Ngaha, 1984 ; Regnoult, 1986 ; Nguetchoua, 1996), et représentent un système aquifère multicouche à nappe gréseuse, sableuse et/ou sablo-argileuse. Cette nappe joue un rôle important dans l'approvisionnement en eau des citadins. Les sols sont dominés par les sols ferrallitiques, les sols hydromorphes, les sols peu évolués et les sols minéraux bruts. Leur teneur en bases est faible et leur $\mathrm{pH}$ acide, de l'ordre de 5,5 (Hieng, 2003).

\section{Echantillonnage}

Deux campagnes d'échantillonnage (de $24 \mathrm{~h}$ ) ont eu lieu respectivement du 16 au 17 février 2008 et du 13 au 14 mars 2008. L'échantillonnage s'est effectué sur le réseau de surface et dans la nappe (Figure 3). Il a consisté en 7 prélèvements (SP1 à SP7) sur le réseau de surface. SP2, SP3, SP6 et SP7 sont situés respectivement d'amont en aval le long du drain principal, le cours d'eau Mgoua. SP1 est situé sur un affluent en amont qui draine une décharge sauvage, SP4 est situé sur l'effluent en provenance de la société CCC (Complexe Chimique Camerounais) tandis que SP5 est localisé sur l'affluent en provenance des brasseries. SP6 et SP7 sont situés à l'aval et devraient par conséquent subir les effets des rejets industriels. Parallèlement, huit points ont été échantillonnés dans la nappe (P1 à P8), distribués dans la zone d'étude. P4 et P8 sont des sources naturelles exploitées par les populations riveraines.

A chaque point, les paramètres physiques (température, $\mathrm{pH}$, conductivité) ont été mesurés sur le terrain à l'aide d'une sonde combinée. Un volume d'eau de deux litres a été prélevé dans des bouteilles plastiques pour analyses physiques complémentaires. Deux échantillons de $60 \mathrm{cl}$, dont un acidifié, ont été stockés pour des besoins d'analyses physicochimiques au laboratoire.

\section{Méthodes d'analyses de laboratoire}

Les analyses ont été effectuées dans le laboratoire PROTEE (PROcessus de Transfert et d'Echanges dans l'Environnement) de l'Université du Sud Toulon-Var en France. En raison de leur caractère incomplet, les analyses chimiques ne seront pas présentées dans cette étude.

L'alcalinité (TAC) a été déterminée par dosage acido-basique contrôlé par un procédé colorimétrique (virage de l'hélianthine à $\mathrm{pH}$ 4,3).

La turbidité a été mesurée dans des néphélomètres par comparaison de la lumière diffusée et de la lumière transmise, d'une part, par l'échantillon testé et, d'autre part, par une gamme étalon constituée de solutions de formazine, sous une radiation incidente de longueur d'onde donnée (norme NF-T-90033).

Le carbone organique (dissout et particulaire) a été analysé par l'intermédiaire de l'analyseur de carbone TOC-5000A (Shimatzu) couplé à un passeur d'échantillons (Durrieu, 2004). La fluorescence 3D obtenue à partir de l'appareil HITACHI Fluorescence Spectrophotomètre F-4500 a permis de déterminer la teneur en carbone organique total (COT). Par la suite, l'utilisation d'un programme de déconvolution numérique (PARAFAC - PARAllel FACtor analysis), en se basant sur la localisation spectrale des différents fluophores, a permis d'identifier les différents composés parmi lesquels les acides fulviques (AFV), le tryptophane et les dérivés protéiques (TDP), les acides humiques marins (AHM), ainsi que les acides humiques aromatiques et le dérivés de la lignine (AHA) (Coble, 1995 ; Chen, 2003 ; Zhao, 2011). Le carbone organique particulaire (COP) a été analysé uniquement dans les eaux de surface.

Les métaux lourds (cuivre, nickel, plomb) ont été dosés par la spectroscopie d'absorption atomique (ThermoElectron GF95Z). 
L. KENGNI et al. / Int. J. Biol. Chem. Sci. 6(4): 1838-1853, 2012

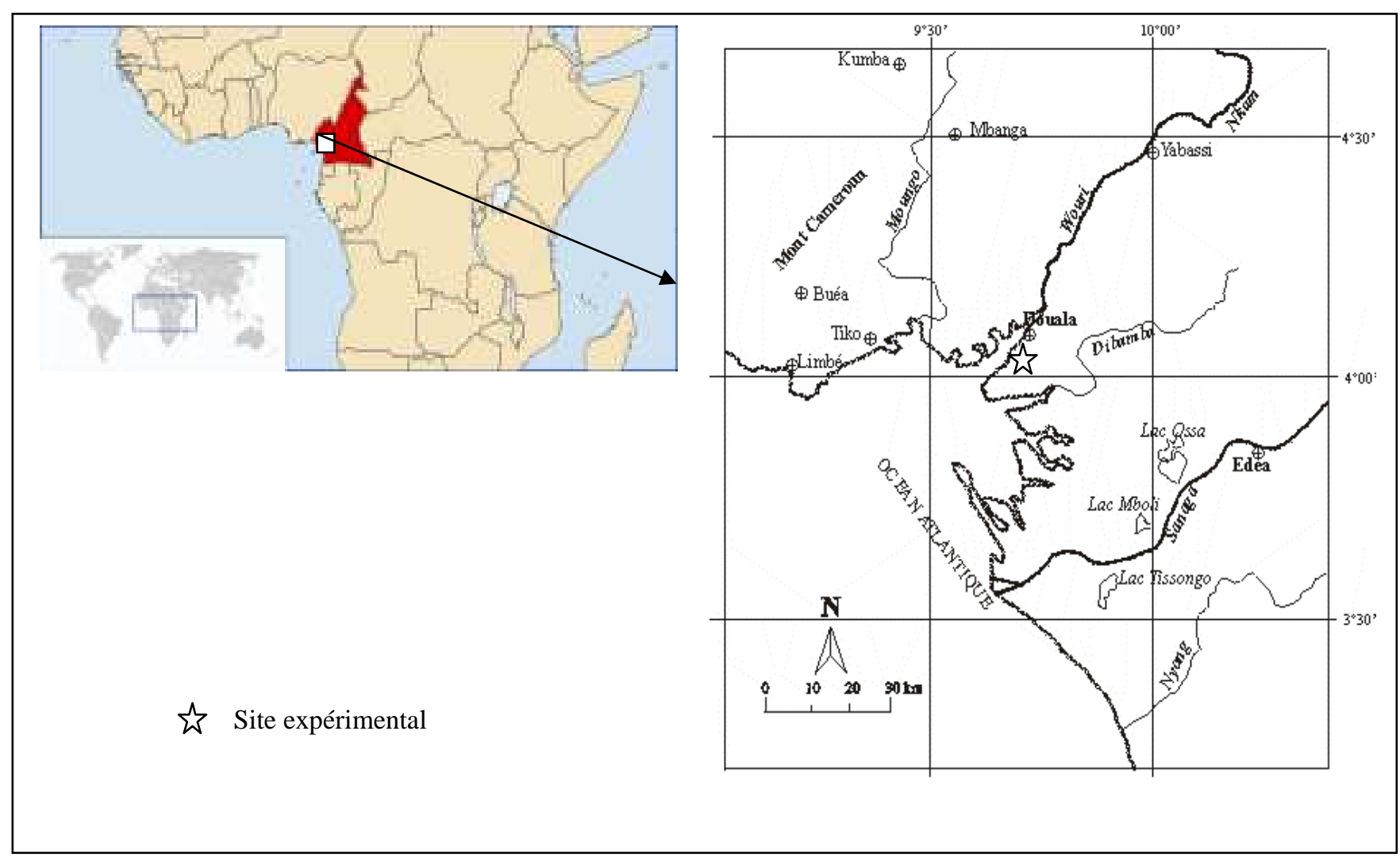

Figure 1: Situation de Douala et du site expérimental. 


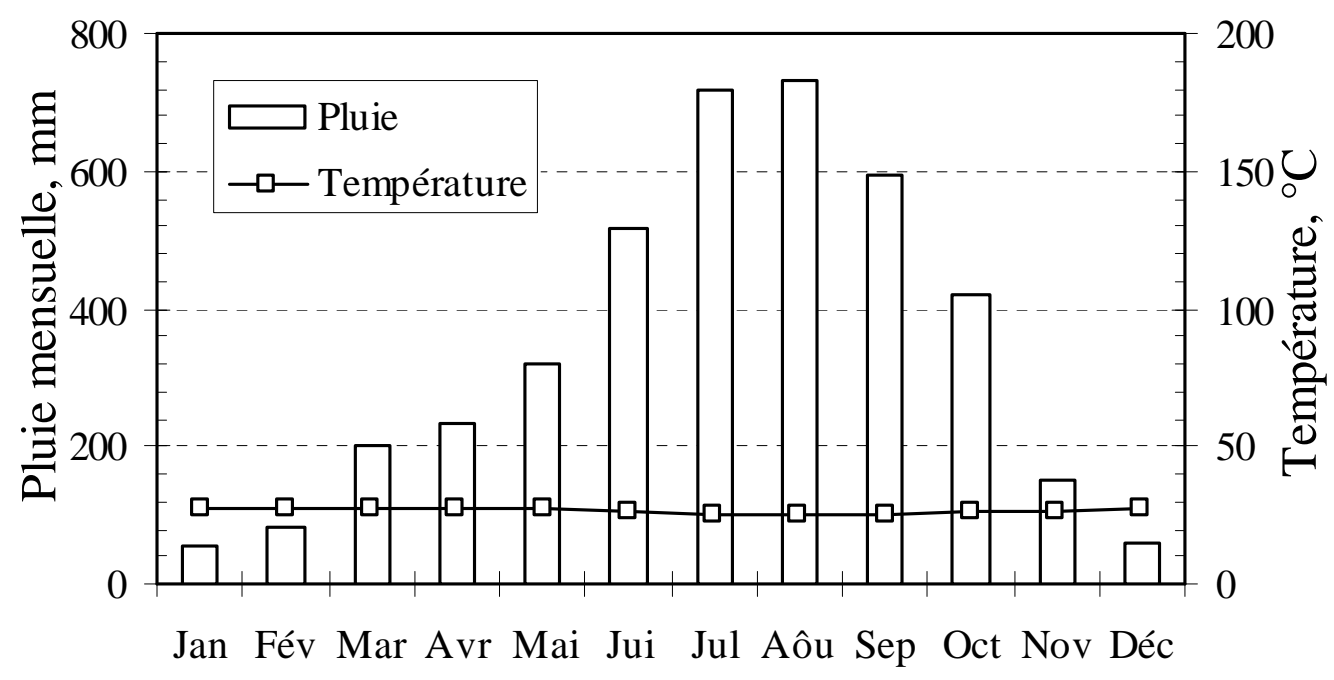

Figure 2: Courbe ombrothermique de Douala.

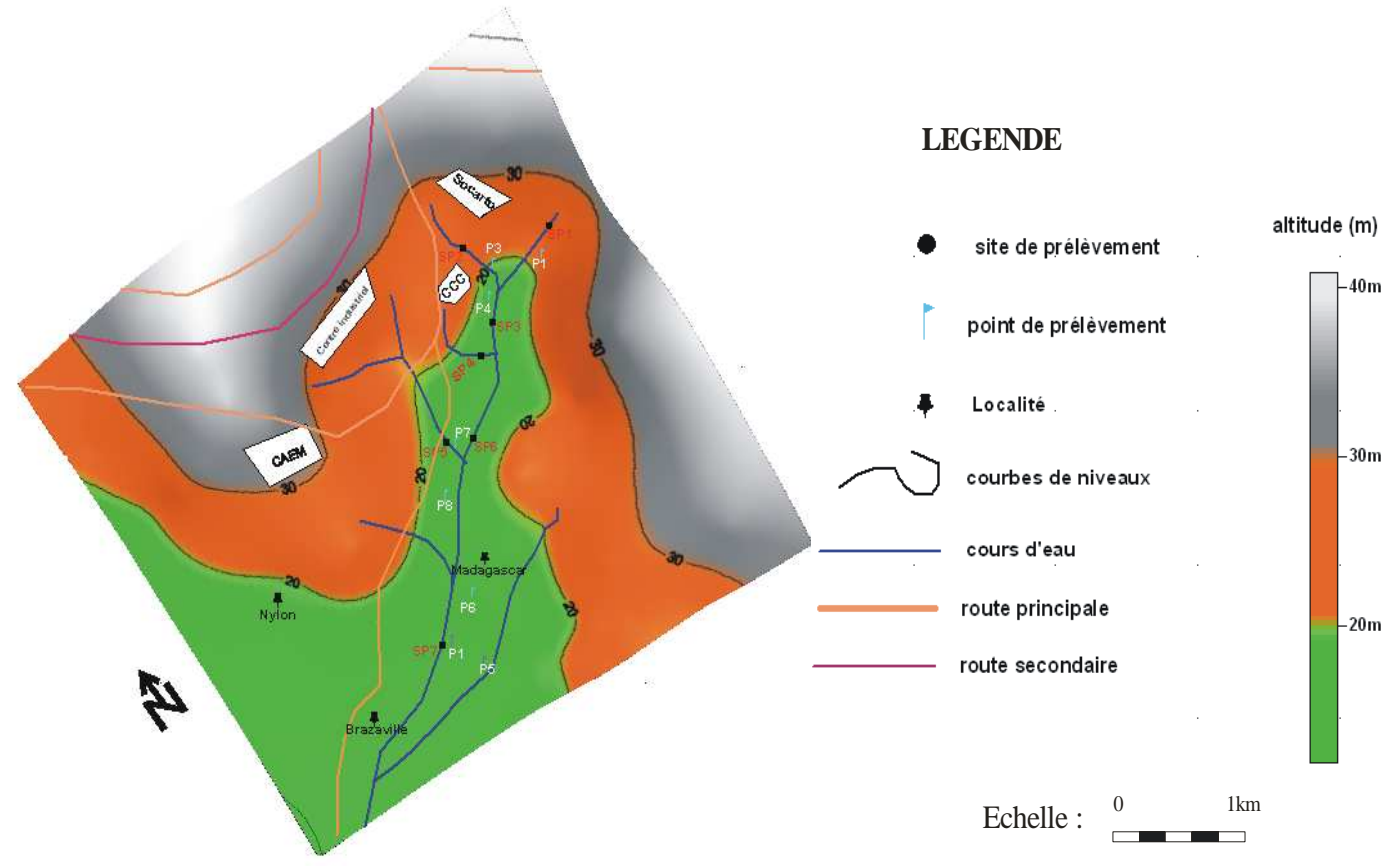

Figure 3: Carte du basin versant et position des sites de prélèvements. 


\section{RESULTATS}

Quelque soit l'élément considéré, il n'a pas été noté de différence significative (au seuil de 5\%) de teneur entre les deux campagnes de prélèvement.

\section{Caractéristiques physiques des eaux de surface}

Les prélèvements pluri-journaliers dans le réseau de surface montrent que les paramètres physiques varient dans la journée (Tableau 1). La température dans chaque site est plus élevée entre $13 \mathrm{~h}$ et $15 \mathrm{~h}$. A l'échelle de la journée, elle varie relativement peu autour de $28,0{ }^{\circ} \mathrm{C}$ (de $30,0{ }^{\circ} \mathrm{C}$ à $27,2{ }^{\circ} \mathrm{C}$ ). Cependant, elle reste supérieure à $30,0{ }^{\circ} \mathrm{C}$ à SP4 où le maximum est plutôt relevé dans la nuit. Le pH est proche de la neutralité $(6,06$ à 6,55). L'effluent du complexe chimique est très basique (pH de 9,31 à 11,40), associé à une conductivité très élevée (1561 à 5890 $\mu \mathrm{S} / \mathrm{cm})$. Les valeurs maximales de turbidité (553 NTU) et de TAC $(40,3 \mathrm{mg} / \mathrm{l})$ sont obtenues également dans le même site.

Ces mêmes tendances s'observent à la deuxième campagne de prélèvement, avec toutefois des amplitudes plus faibles.

\section{Teneur en carbone organique Carbone organique dissout}

A l'échelle de la journée, les teneurs en COD restent faibles au niveau des stations situées en tête de bassin dans le réseau de surface (SP1, SP2 et SP3). Sur l'exemple du 16 février (Figure 4), la valeur maximale est de $3,1 \mathrm{mg} / \mathrm{l}$. La variation temporelle y est également faible. L'effluent en provenance de la savonnerie (SP4) montre cependant des valeurs très élevées ainsi qu'une forte variation temporelle. Le maximum (1732,5 $\mathrm{mg} / \mathrm{l}$ ) est ainsi observé à minuit, moment probable où se font les rejets, et le minimum, $75,0 \mathrm{mg} / \mathrm{l}$, à $13 \mathrm{~h}$. Ces fortes valeurs se répercutent ensuite sur les autres points de prélèvement situés à l'aval (SP6 et SP7).

En valeur moyenne, les teneurs journalières varient selon les sites de 2,0 à $1020,6 \mathrm{mg} / \mathrm{l}$ pour la première campagne et de 3,0 à $571,7 \mathrm{mg} / \mathrm{l}$ pour la deuxième campagne
(Figure 5). Ces concentrations sont faibles de SP1 à SP3 situés à l'amont du bassin où elles ne dépassent pas en valeur moyenne $4,0 \mathrm{mg} / \mathrm{l}$. Par contre, l'effluent en provenance du complexe chimique (SP4) montre des concentrations très élevées, pouvant atteindre $1020,6 \mathrm{mg} / \mathrm{l}$ à la première campagne et 571,7 $\mathrm{mg} / \mathrm{l}$ lors de la deuxième campagne. L'effluent est dilué au fur et à mesure dans le réseau de surface (SP6 et SP7). Ces teneurs baissent de manière significative en aval mais restent sensibles le long du cours d'eau principal. Il est à noter une source moins importante de COD en provenance des brasseries (SP5).

Si l'on s'intéresse aux eaux de nappe, les teneurs en COD restent largement faibles, comparées à celles enregistrées dans les cours d'eau (Figure 6). Ainsi, les puits P2, P3 et P4 ont des valeurs qui ne dépassent guère 1,8 $\mu \mathrm{g} / \mathrm{l}$ en moyenne. En aval (P1), les teneurs maximales s'élèvent à $6,5 \mu \mathrm{g} / \mathrm{l}$. Le site $\mathrm{P} 7 \mathrm{se}$ distingue par des concentrations très élevées, atteignant $14,8 \mu \mathrm{g} / \mathrm{l}$.

\section{Carbone organique particulaire}

Le comportement du carbone organique particulaire (Figure 7) reste similaire à celui du carbone dissout, mais avec une amplitude plus faible: faibles valeurs à l'amont du bassin (de 0,4 à $0,9 \mathrm{mg} / \mathrm{l})$, puis fortes à l'aval où elles n'excèdent pas toutefois $3,3 \mathrm{mg} / \mathrm{l}$. Cette augmentation résulte de l'influence des apports de la savonnerie (de 8,6 à 19,6 mg/l).

\section{Fractionnement de la matière organique}

Les différents composés de la matière organique ont été identifiés sur les échantillons du 16 février uniquement. Dans les cours d'eau, les quatre composés différenciés existent et participent à la fluorescence à des proportions variables, de 0,032 à 1,041 . Ces contributions, faibles à l'amont $(<0,1)$, augmentent à partir de SP4 pour atteindre 0,77 à l'exutoire du bassin. En dehors de SP1, les AHA sont peu représentés par rapport aux autres composés.

Dans les eaux de puits, les contributions varient de 0,003 à 1,338 . Deux groupes semblent se dessiner (Figure 8). Un premier groupe inclut les puits de haut de versant ( $\mathrm{P} 2, \mathrm{P} 3, \mathrm{P} 4, \mathrm{P} 6$ et $\mathrm{P} 8)$ ne contenant 
que trois dérivés : AFV, TRP et AHM. Ces puits sont également caractérisés par une faible intensité de fluorescence, quel que soit le composé, ne dépassant pas 0,1. Le deuxième groupe est formé de $\mathrm{P} 1, \mathrm{P} 5$ et $\mathrm{P} 7$ situés en bas de versant. Le taux de participation varie de 0,003 à $1,338(0,434$ si on exclut P7), avec l'apparition des AHA. P7 contenant le maximum de COD se distingue à nouveau dans ce groupe par un taux de participation très élevé des $\mathrm{AFV}(1,338)$ (contre 1,041 à SP4) et des AHM $(0,414)$.

\section{Teneur en métaux}

Dans les cours d'eau, les éléments $\mathrm{Ni}$, $\mathrm{Cu}$ et $\mathrm{Pb}$ sont rencontrés en proportions très variables (Figure 9). A l'exception du nickel, les concentrations en métaux de la $2^{\mathrm{e}}$ campagne sont supérieures à celles de la $1^{\text {ère }}$ campagne.

Le nickel varie de 27,01 à $34,89 \mu \mathrm{g} / \mathrm{l}$ en février et de 5,43 à 16,24 $\mu \mathrm{g} / 1$ en mars. Aucun site ne se démarque particulièrement.
Le cuivre montre également une variation importante dans les deux prélèvements. Toutefois, les teneurs maximales ne dépassent pas 19,44 $\mu \mathrm{g} / \mathrm{l}$.

Les teneurs en plomb sont extrêmement variables, de 1,12 à $12,24 \mu \mathrm{g} / \mathrm{l}$ lors de la première campagne et de 2,01 à 20,33 $\mu \mathrm{g} / \mathrm{l}$ pendant la deuxième série. SP2 pourtant en tête de bassin (proche de SOCARTO) montre des concentrations plus élevées qu'à l'exutoire (SP7) pendant la deuxième campagne.

Dans les nappes, les teneurs en métaux sont plus faibles par rapport à celles des cours d'eau (Figure 10). La teneur en $\mathrm{Cu}$ varie peu, de 6,83 à 10,63 $\mu \mathrm{g} / \mathrm{l}$. Cet élément montre en même temps peu d'écart par rapport aux eaux de surface (de 4,48 à 19,44 $\mu \mathrm{g} / \mathrm{l}$ ). Les teneurs en $\mathrm{Ni}$ sont plus variables, P7 a la concentration la plus élevée, $15 \mu \mathrm{g} / \mathrm{l}$. Le $\mathrm{Pb}$ varie également peu, de 1,8 à 9,6 $\mu \mathrm{g} / \mathrm{l}$.

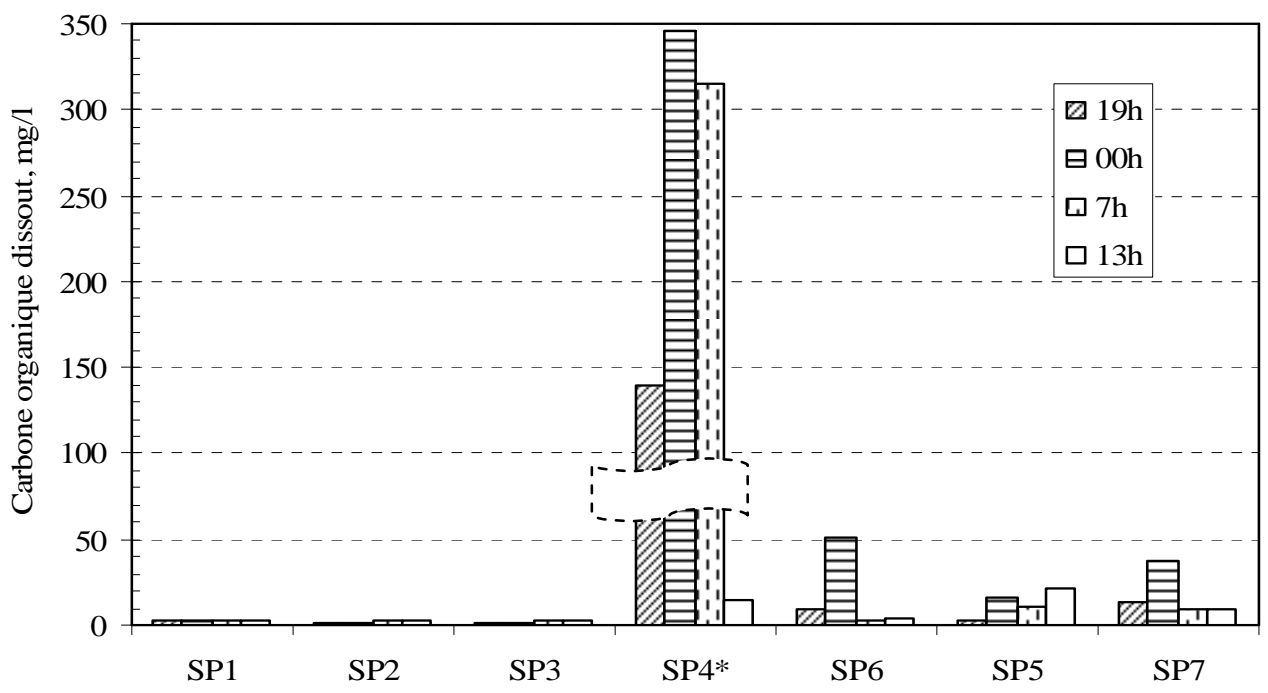

Figure 4: Evolution journalière du COD du 16 au 17 février dans les eaux de surface (Les valeurs de SP4 ont été divisées par 5 pour les besoins du graphique). 


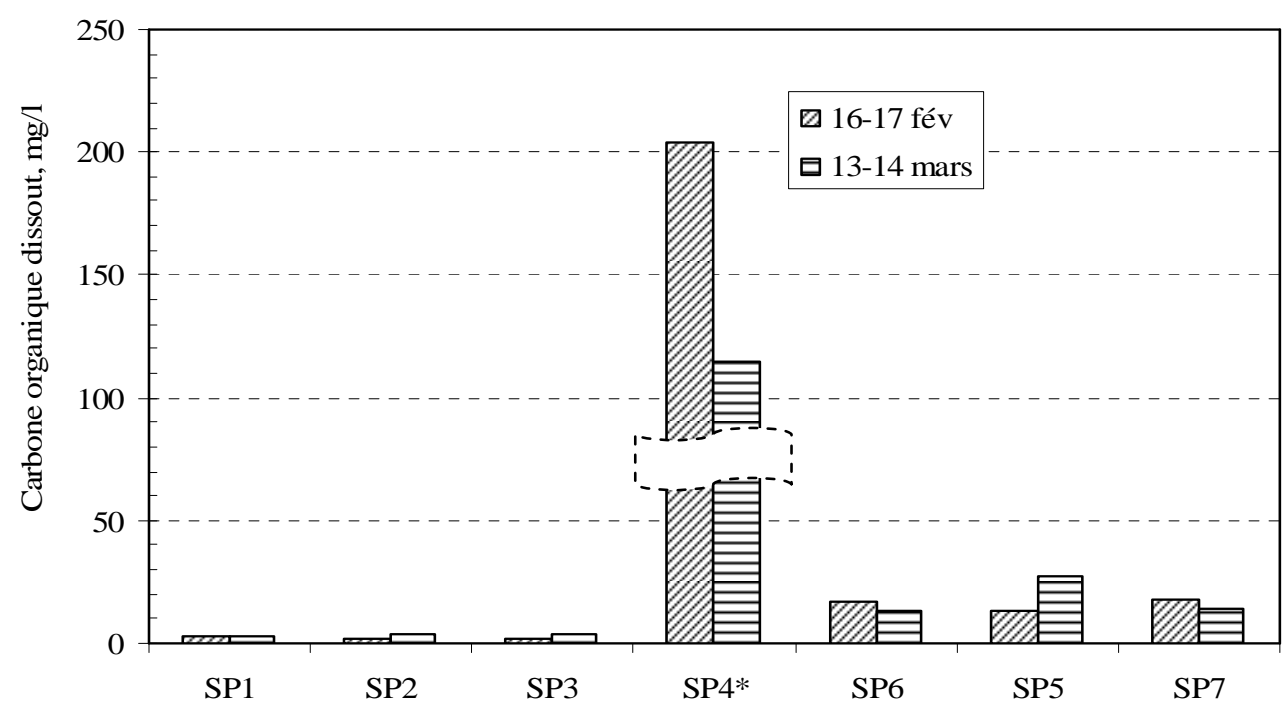

Figure 5: Teneur moyenne du COD dans les eaux de surface (Les valeurs de SP4 ont été divisées par 5 pour les besoins du graphique).

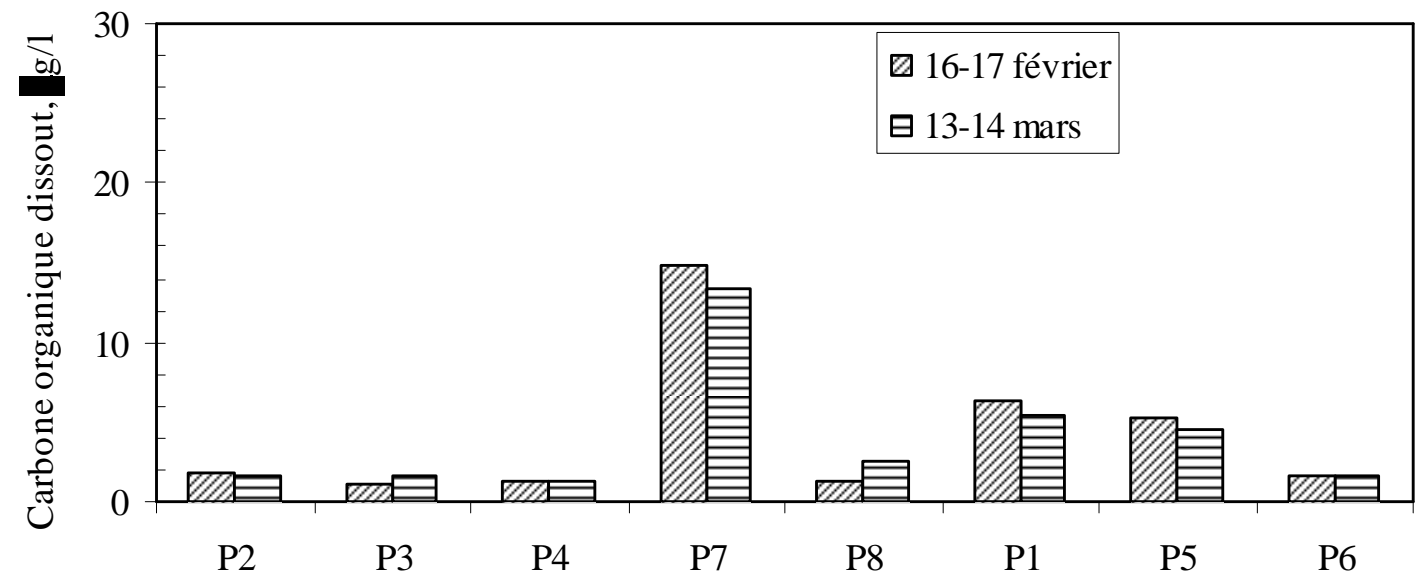

Figure 6: Teneur moyenne du COD dans les puits. 


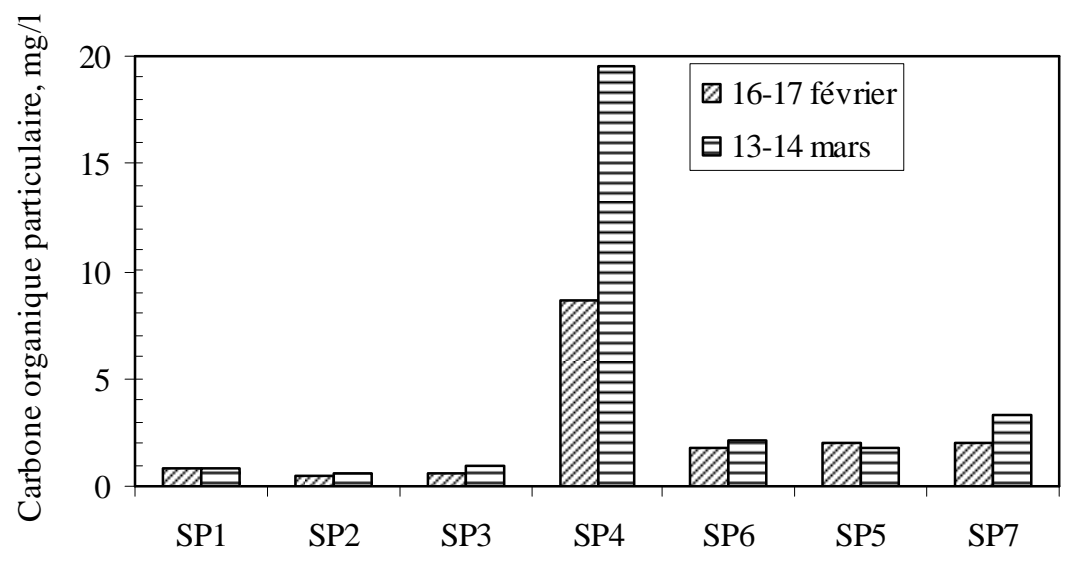

Figure 7: Teneur moyenne du COP dans les eaux de surface.
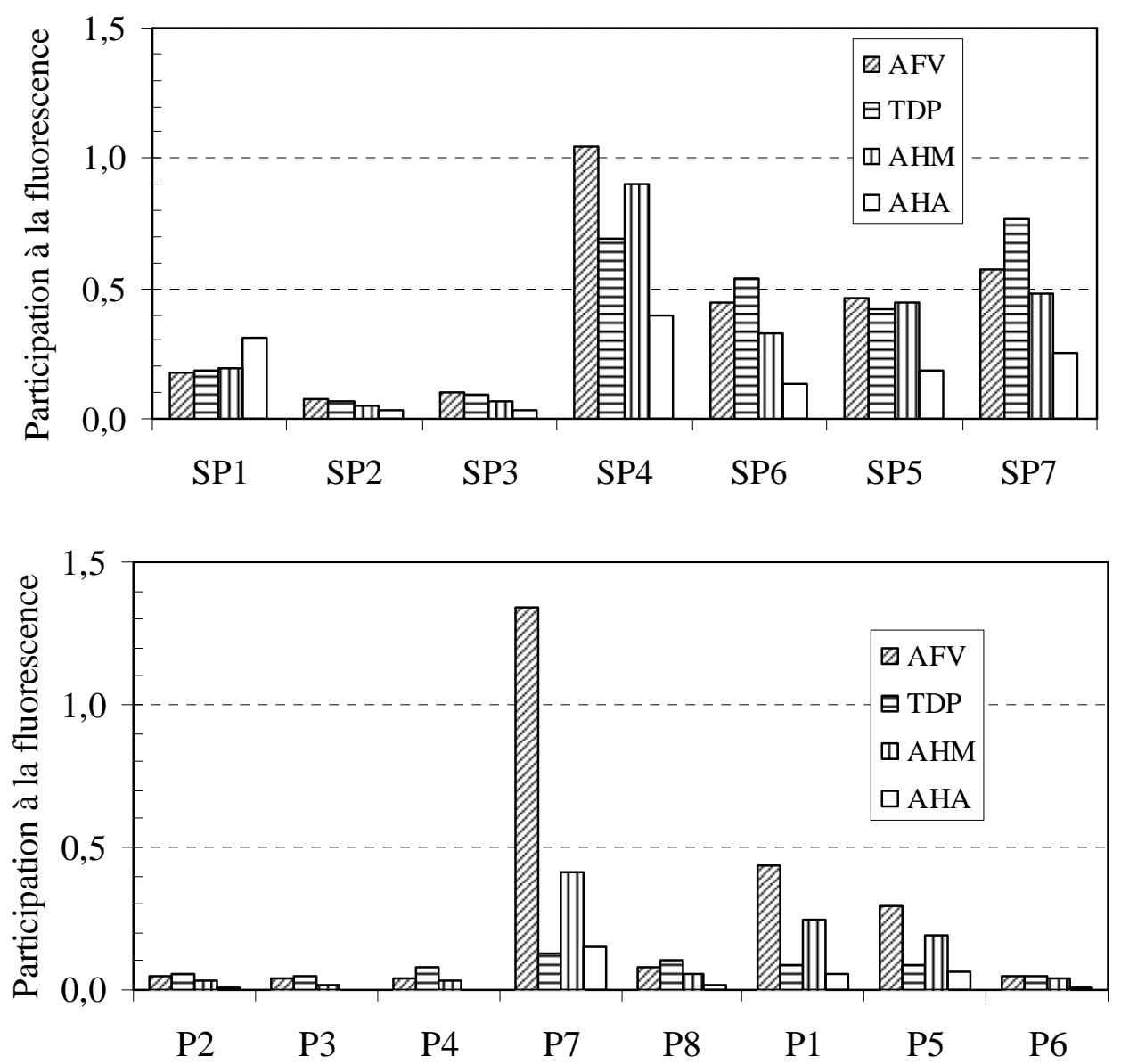

Figure 8: Composés de la matière organique dans les eaux de surface et des puits. AFV : Acides fulviques; TDP : Tryptophane et dérivés protéiques; AHM : Acides humiques marins; AHA : Acides humiques aromatiques et dérivés de la lignine. 


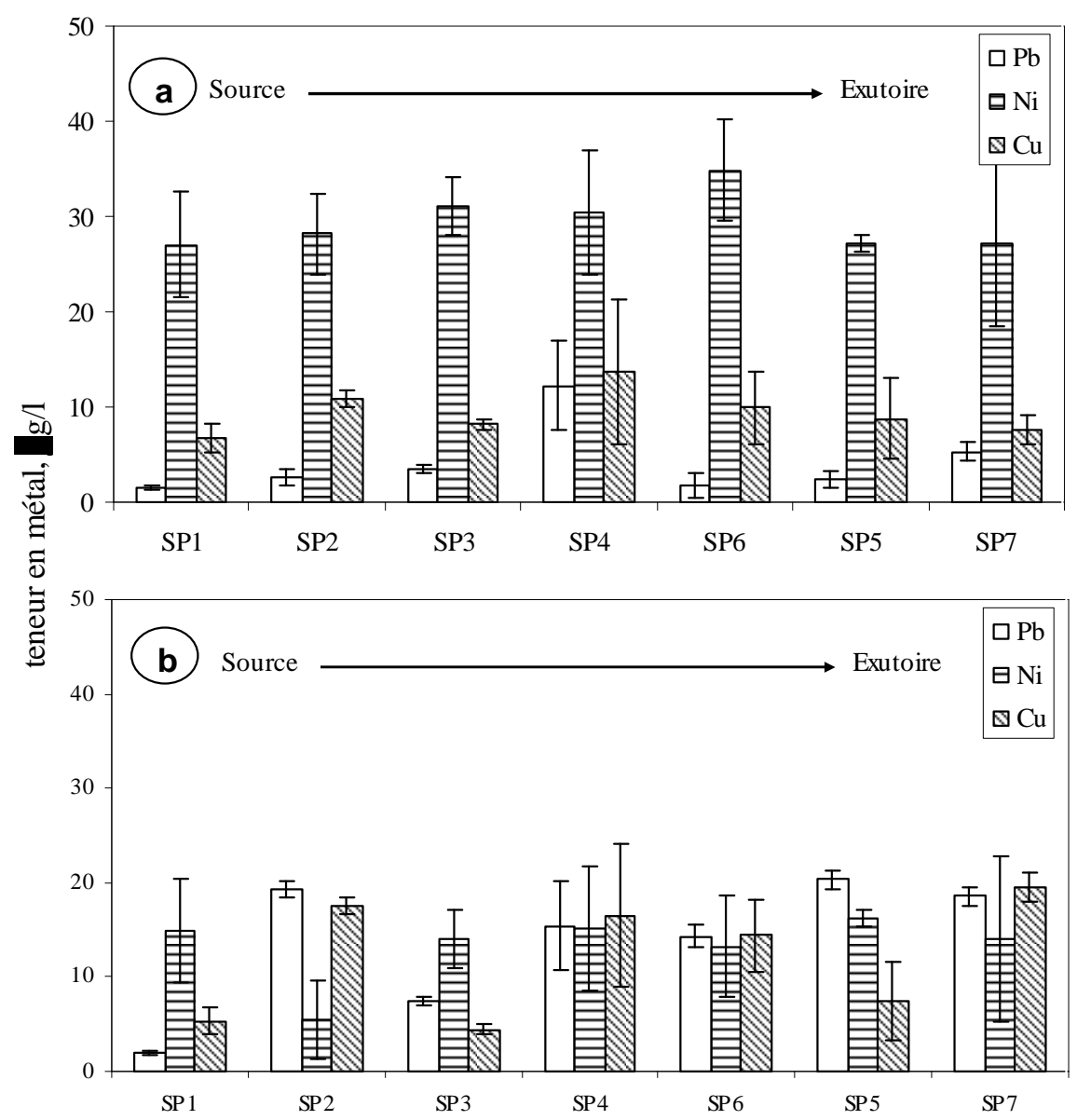

Figure 9: Teneur moyenne en métaux dans le réseau de surface : a) 16-17 février, b) 13-14 mars.

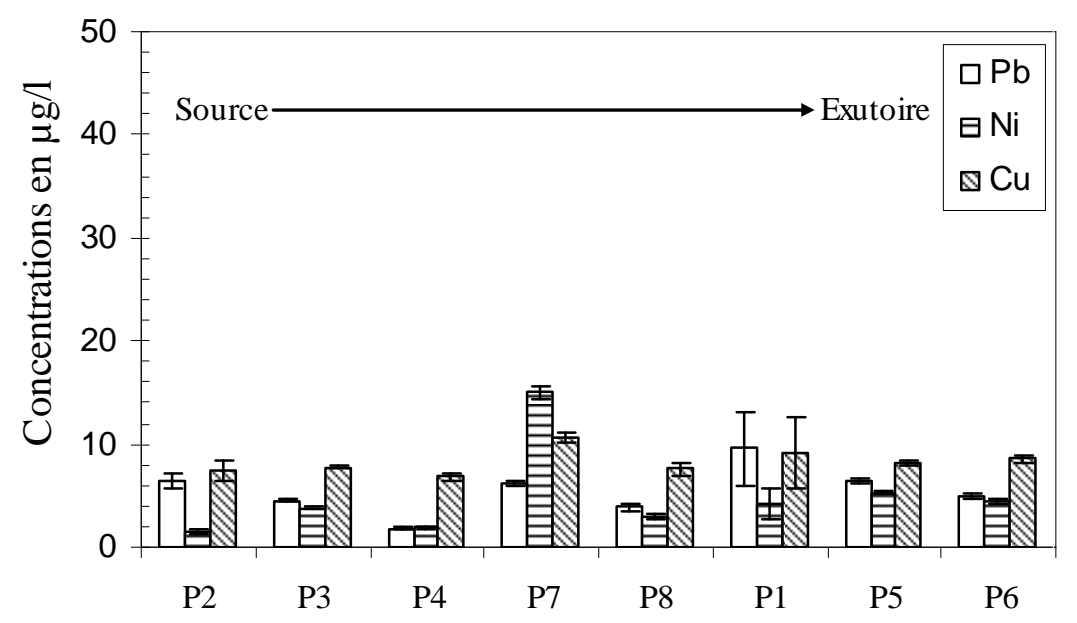

Figure 10: Teneur moyenne en métaux dans les puits. 


\section{DISCUSSION}

Les variations des teneurs en éléments dans le réseau de surface témoignent d'une contamination irrégulière et parfois importante. Dans le bassin, les eaux de surface sont neutres à l'amont ( $\mathrm{pH}$ 6,2), peu minéralisées (conductivité moyenne 262 $\mu \mathrm{S} / \mathrm{cm}$ ) et peu turbides (7 NTU). SP4, l'effluent de la savonnerie, se distingue par son caractère très basique $(\mathrm{pH}$ de 9,31 à 11,40), sa forte minéralisation (conductivité de 1651 à $5890 \mu \mathrm{S} / \mathrm{cm}$ ) et son alcalinité élevée $(40,3 \mathrm{mg} / \mathrm{l})$. L'effluent en provenance des brasseries montre une tendance acide $(\mathrm{pH}$ de 5,8 à 6,4 ). On note également une bonne corrélation entre la conductivité et les bicarbonates $(R=0,91)$. Cet élément domine la conductivité comme le montrent les échantillons du 16 février (Figure 11) prélevés en conditions d'écoulement naturel (2 semaines après une pluie). Vers l'aval du bassin, les eaux deviennent nettement acides, de $\mathrm{pH}$ de l'ordre de 5,9 à partir de SP6. Ce caractère résulte de l'augmentation du taux de matières organiques dont le COT variant en moyenne de 2 à $4 \mathrm{mg} / \mathrm{l}$ à l'amont passe audelà de $13 \mathrm{mg} / \mathrm{l}$ à l'aval, corrélativement à l'apparition des AHA (Meyer, 1990 cité par Cotrim Da Cunha, 2000). L'augmentation de l'acidité provoque la libération et le lessivage des cations adsorbés sur les matières en suspension, d'où l'augmentation de la conductivité. L'activité industrielle (SP4) génère par conséquent une importante charge (en matières organiques et en bicarbonates) qui se reflète dans la turbidité et la conductivité de l'eau. Ces dernières valeurs moyennes passent respectivement de 7 à 168 NTU et de 198 à $796 \mu \mathrm{S} / \mathrm{cm}$. Ces apports en provenance de la savonnerie accentuent également la teneur en COT essentiellement sous forme dissoute. La MO est transportée essentiellement sous forme dissoute, le rapport $\mathrm{COD} / \mathrm{COP}$ varie en effet de 2,7 à 119,0 (de 2,7 à 15,2 si on exclut SP4). L'absence de traitement préalable serait en grande partie responsable de cette pollution, de même que les lixiviats de la décharge publique.

La corrélation est également forte entre les carbonates et la matière organique $(\mathrm{R}=$ 0,98 ) (Figure 11). Le processus d'évolution de la matière organique est par conséquent la minéralisation, suivie d'une libération d'éléments entre autre des carbonates. La conductivité élevée (associée à une température élevée) dénote donc d'une minéralisation excessive attribuable aux rejets domestiques ou industriels déversés. A l'exutoire du bassin apparaissent les AHA (acides humiques aromatiques et les dérivés de la lignine) qui témoignent du caractère acide des eaux (Cotrim Da Cunha, 2000).
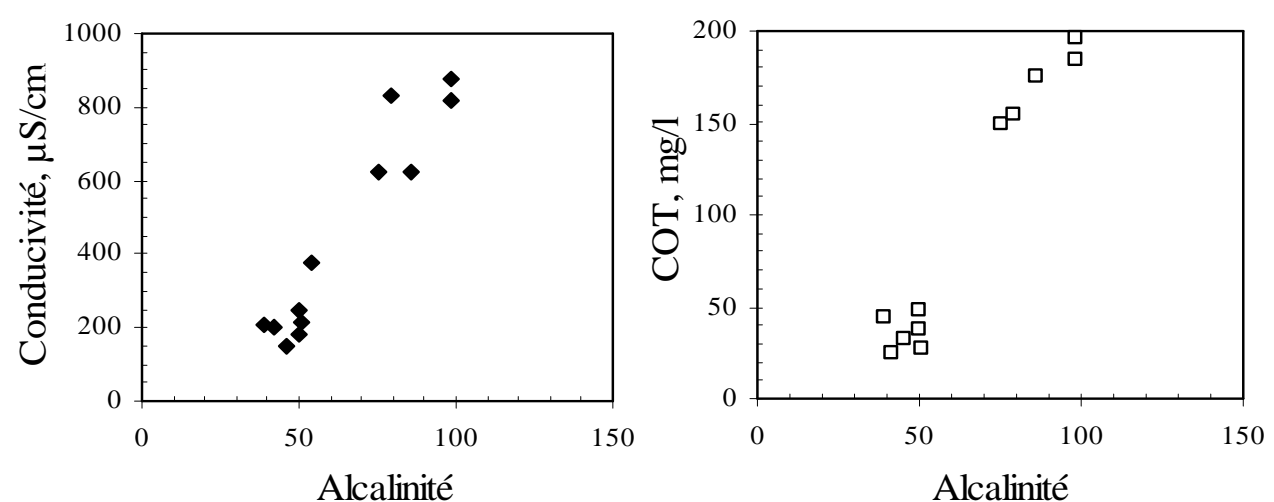

Figure 11: Relation entre la teneur en carbonates et la conductivité ou le carbone organique total. 
Tableau 1: Caractéristiques physiques des eaux de surface.

\begin{tabular}{|c|c|c|c|c|c|c|c|c|c|c|c|c|c|}
\hline \multicolumn{8}{|c|}{ Prélèvement du 16 au 17 Février 2008} & \multicolumn{6}{|c|}{ Prélèvement du 13 au 14 Mars 2008} \\
\hline Site & heure & $\begin{array}{c}\mathbf{T} \\
\left({ }^{\circ} \mathbf{C}\right) \\
\end{array}$ & pH & $\begin{array}{c}\text { Cond } \\
(\mu \mathrm{S} / \mathrm{cm})\end{array}$ & $\begin{array}{c}\text { Turb } \\
\text { (NTU) }\end{array}$ & $\begin{array}{l}\text { TAC } \\
\left({ }^{\circ} \mathbf{F}\right)\end{array}$ & Site & heure & $\begin{array}{c}\mathbf{T} \\
\left({ }^{\circ} \mathbf{C}\right) \\
\end{array}$ & $\mathbf{p H}$ & $\begin{array}{c}\text { Cond } \\
(\mu \mathrm{S} / \mathrm{cm})\end{array}$ & $\begin{array}{l}\text { Turb) } \\
\text { (NTU) }\end{array}$ & $\begin{array}{l}\text { TAC } \\
\left({ }^{\circ} \mathbf{F}\right)\end{array}$ \\
\hline SP1 & $19 \mathrm{~h} 15$ & 28,2 & 6,47 & 175 & 15 & 4,65 & SP1 & $15 \mathrm{~h} 00$ & 30,4 & 6,51 & 176 & 9 & 4,35 \\
\hline SP1 & 00h10 & 27,3 & 6,55 & 124 & 7 & 3,20 & SP1 & $23 \mathrm{~h} 00$ & 27,8 & 6,72 & 172 & 13 & 3,80 \\
\hline SP1 & $07 \mathrm{~h} 27$ & 25,6 & 6,48 & 142 & 12 & 3,70 & SP1 & $7 \mathrm{~h} 30$ & 26,8 & 6,53 & 198 & 9 & 4,17 \\
\hline SP1 & $13 \mathrm{~h} 11$ & 29,8 & 6,50 & 146 & 8 & 3,45 & SP2 & $15 \mathrm{~h} 56$ & 30,4 & 6,21 & 217 & 7 & 3,51 \\
\hline SP2 & $19 \mathrm{~h} 25$ & 27,8 & 6,06 & 205 & 2 & 3,05 & SP2 & $23 \mathrm{~h} 10$ & 27,7 & 6,46 & 193 & 3 & 2,94 \\
\hline SP2 & $00 \mathrm{~h} 25$ & 27,3 & 6,06 & 203 & 2 & 3,40 & $\mathrm{SP} 2$ & $8 \mathrm{~h} 05$ & 27,3 & 6,54 & 209 & 9 & 3,19 \\
\hline $\mathrm{SP} 2$ & $08 \mathrm{~h} 00$ & 27,4 & 6,08 & 209 & 2 & 3,60 & SP3 & $16 \mathrm{~h} 10$ & 30,4 & 6,40 & 258 & 10 & 4,01 \\
\hline SP2 & $13 \mathrm{~h} 28$ & 30,1 & 6,15 & 198 & 3 & 3,65 & SP3 & $23 \mathrm{~h} 25$ & 27,3 & 6,71 & 230 & 9 & 4,21 \\
\hline SP3 & $19 \mathrm{~h} 38$ & 28,0 & 6,38 & 212 & 5 & 3,90 & SP3 & $8 \mathrm{~h} 32$ & 27,3 & 6,69 & 260 & 6 & 3,99 \\
\hline SP3 & 00h32 & 27,9 & 6,33 & 215 & 5 & 4,15 & SP4 & $16 \mathrm{~h} 30$ & 31,2 & $\mathbf{9 , 5 2}$ & 1946 & 370 & 18,91 \\
\hline SP3 & 08h17 & 27,2 & 6,40 & 209 & 7 & 4,25 & SP4 & $23 h 35$ & 29,0 & $\mathbf{9 , 1 7}$ & 1434 & 474 & 33,13 \\
\hline SP3 & $13 \mathrm{~h} 40$ & 31,4 & 6,41 & 212 & 6 & 4,30 & SP4 & $8 h 43$ & 29,6 & 8,24 & 997 & 427 & $\mathbf{3 5 , 8 9}$ \\
\hline SP4 & $20 \mathrm{~h} 00$ & 30,6 & $\mathbf{9 , 3 1}$ & 1561 & 324 & 16,75 & SP5 & $17 \mathrm{~h} 24$ & 30,9 & 5,11 & 413 & 147 & 5,01 \\
\hline SP4 & $00 h 50$ & 35,2 & 11,15 & 5890 & 553 & 35,75 & SP5 & $23 \mathrm{~h} 55$ & 30,0 & 5,90 & 350 & 117 & 4,35 \\
\hline SP4 & 08h53 & 30,0 & 11,40 & 4550 & 378 & 40,25 & SP5 & $9 \mathrm{~h} 28$ & 31,6 & 6,40 & 369 & 121 & 3,91 \\
\hline SP4 & $13 \mathrm{~h} 53$ & 32,3 & 10,09 & 1856 & 329 & 18,15 & SP6 & $17 \mathrm{~h} 20$ & 29,8 & 5,82 & 954 & 198 & 6,18 \\
\hline
\end{tabular}


L. KENGNI et al. / Int. J. Biol. Chem. Sci. 6(4): 1838-1853, 2012

\begin{tabular}{cccccccccccccc} 
SP5 & $20 \mathrm{~h} 35$ & 28,5 & 6,60 & 509 & 9 & 5,65 & SP6 & $00 \mathrm{~h} 00$ & 27,9 & 6,17 & 909 & 187 & 5,13 \\
SP5 & $01 \mathrm{~h} 15$ & 27,8 & 6,56 & 566 & 8 & 4,85 & SP6 & $9 \mathrm{~h} 14$ & 28,5 & 6,33 & 629 & 147 & 8,13 \\
SP5 & $09 \mathrm{~h} 12$ & 28,3 & 6,60 & 598 & 7 & 4,85 & SP7 & $18 \mathrm{~h} 23$ & 30,9 & 5,64 & 717 & 213 & 6,59 \\
SP5 & $14 \mathrm{~h} 07$ & 30,3 & 5,85 & 825 & 194 & 9,25 & SP7 & $00 \mathrm{~h} 15$ & 28,5 & 5,68 & 587 & 267 & 7,02 \\
SP6 & $21 \mathrm{~h} 40$ & 28,5 & 5,80 & 505 & 90 & 6,90 & SP7 & $11 \mathrm{~h} 07$ & 31,8 & 6,22 & 566 & 181 & 7,51 \\
SP6 & $01 \mathrm{~h} 25$ & 28,6 & 4,99 & 1307 & 329 & 7,75 & & & & & & & \\
SP6 & $09 \mathrm{~h} 25$ & 29,1 & 5,84 & 510 & 95 & 6,85 & & & & & & \\
SP6 & $14 \mathrm{~h} 20$ & 30,2 & 6,99 & 1195 & 15 & 10,80 & & & & & & \\
SP7 & $22 \mathrm{~h} 10$ & 28,7 & 5,94 & 631 & 63 & 7,35 & & & & & & \\
SP7 & $01 \mathrm{~h} 35$ & 28,1 & 5,86 & 784 & 75 & 6,85 & & & & & & \\
SP7 & $09 \mathrm{~h} 45$ & 28,6 & 5,96 & 577 & 260 & 6,20 & & & & & & \\
SP7 & $14 \mathrm{~h} 49$ & 33,2 & 5,73 & 1280 & 236 & 11,80 & & & & & & & \\
& & & & & & & & & & \\
Minimum & 25,6 & 4,99 & 124 & 2 & 3,05 & & Minimum & 26,8 & 5,11 & 172 & 3 & 2,94 \\
Moyenne & 29,1 & 6,81 & 907 & 109 & 8,76 & Moyenne & 29,3 & 6,64 & 548 & 135 & 8,50 \\
Maximum & 35,2 & 11,40 & 5890 & 553 & 40,25 & Maximum & 31,8 & 9,52 & 1946 & 474 & 35,89 \\
Ecart-type & 2,0 & 1,61 & 1318 & 153 & 9,12 & Ecart-type & 1,6 & 1,09 & 468 & 147 & 9,33 \\
Amplitude & 9,6 & 6,41 & 5766 & 551 & 37,20 & Amplitude & 5,0 & 4,41 & 1774 & 471 & 32,95 \\
\hline
\end{tabular}

Cond : conductivité ; Turb : turbidité ; TAC : titre alcalinimétrique complet En gras : Valeurs relevées sur le site de l'effluent industriel. 
Le comportement des métaux est mitigé dans les eaux de surface qui sont par ailleurs polluées d'après les critères de Merian (1996). Les concentrations en $\mathrm{Cu}$ et $\mathrm{Ni}$ restent systématiquement inférieures à la norme de l'OMS (2006) pour les eaux potables (respectivement de 2000 et $70 \mu \mathrm{g} / \mathrm{l}$ ). Par contre, les concentrations en $\mathrm{Pb}$ pour les différents échantillons sont parfois supérieures à la limite admise pour les eaux potables (10 $\mu \mathrm{g} / \mathrm{l})$. Aucun site ne se distingue particulièrement, ni même SP4. Ces diverses substances sont véhiculées par les eaux météoriques, les eaux de ruissellement des toitures et voiries sans oublier les eaux de ruissellement des chaussées (Colandini, 1997 ; Durand, 2003). Par conséquent, une pollution d'origine industrielle ne peut être incriminée. Les sols camerounais renferment en moyenne 28,76 $\mu \mathrm{g} / \mathrm{l}$ de plomb (Tchamegni, 1997).

Dans les eaux de nappe, les teneurs en métaux sont plus faibles, en regard des concentrations dans les eaux de surface. Ce bilan positif des métaux lourds militent dans un scénario de stockage notamment dans le sol et les berges des cours d'eau. Ceci met en évidence le rôle protecteur des berges des cours d'eau et de la zone non saturée. De nombreuses études font état du piégeage des métaux dans les premiers centimètres du sol, en particulier sur la matière organique (Alloway, 1995 ; Alloway et Jackson, 1991 ; Bacon et al., 1995 ; Baque, 2006 ; Devallois, 2009). Toutefois, il se dégage que les concentrations maximales en $\mathrm{Ni}$ sont observées dans la moitié aval du bassin. Le puits P7 montre par ailleurs la teneur la plus élevée $(15 \mu \mathrm{g} / \mathrm{l})$, en même temps qu'un maximum de COD $(14,8 \mu \mathrm{g} / \mathrm{l})$. Le plomb atteint la valeur limite au puits $\mathrm{P} 1$. Il en résulte une source potentielle de pollution des eaux souterraines au nickel, au plomb et en carbone organique à partir des versants. Cette pollution vient s'ajouter à celles chimiques et biologiques mises en évidence par d'autres auteurs dans l'agglomération de Douala (Assonfack, 2002 ; Ketchemen-Tandia et al., 2004 ; Baok, 2007 ; Ndjama et al., 2008 ; Djuikom et al., 2009 ; Temgoua et al., 2009 ).
On note à l'état actuel une absence de contamination critique des eaux souterraines. Si les concentrations actuellement enregistrées n'incitent cependant pas à des inquiétudes immédiates, le risque toxicologique réside dans le caractère cumulatif des métaux lourds. Les dépôts vaseux situés au fond des cours d'eau et latéralement à proximité des berges sont le siège entre autre processus de l'accumulation des métaux lourds qui se fixent sur les particules argileuses des alluvions. En l'absence de l'étude des sols, des sédiments des cours d'eau et des interactions entre la matière organique et les métaux lourds, les phénomènes de rétention ou relargage dans le sol ou le lit des cours d'eau ne peuvent pas être élucidés (Colandini, 1997 ; Gossart, 2001 ; Durand, 2003).

\section{Conclusion}

Les résultats de l'étude montrent quelques aspects de la pollution des eaux en milieu urbain. Les eaux de surface sont contaminées par l'intensification des activités anthropiques, résultant de l'urbanisation et de l'industrialisation. Les polluants sont présents à des concentrations d'importance variable d'un site de prélèvement à l'autre. C'est ainsi que les eaux de surface, neutres, peu minéralisées et peu turbides à l'amont du bassin deviennent acides, très minéralisées et turbides à l'aval. On a noté également une augmentation du taux de matières organiques, due à l'activité industrielle. La charge en métaux est variable. Les eaux souterraines montrent des indices de pollution en métaux lourds et en carbone organique. Les concentrations actuellement enregistrées n'incitent pas à des inquiétudes immédiates. Toutefois, cette pollution des eaux souterraines peut compromettre l'exploitation de la ressource pour de longues périodes de temps. La contamination de ces eaux se faisant par infiltration, il est important d'étudier le comportement des sols et des polluants en terme de rétention, de vitesse de migration ou de compétition entre les différents métaux. 


\section{REFERENCES}

Alloway BJ. 1995. The origin of heavy metals in soils. In Heavy Metals in Soils (2 edn), Alloway BJ (ed). Blackie Academic and Professional: Glasgow; 38-55.

Alloway BJ, Jackson AP. 1991. The behavior of heavy metals in sewage sludge amended soils. Sci. Total Env., 100 : 151176.

Assonfack E. 2002. Contribution à l'étude hydrogéologique de la nappe phréatique de Douala: Caractérisation physicochimique et piézométrique de la nappe à Bassa. Mémoire de Maîtrise, Département de Géologie, Univ. Douala, $49 \mathrm{p}$.

Bacon JR , Berrow ML, Shand CA. 1995. The use of isotopic composition in fields of lead in upland Scottishsoils (U.K.). Chem. Geol., 124: 125-134.

Baok G. 2007. Pollution des eaux de rivière et les impacts biologiques sur les populations riveraines : cas de la rivière Mgoua dans la zone industrielle de Douala-Bassa (Cameroun). Mémoire Master of Science en Gestion de l'Eau, Univ. Dschang, 96p.

Baque D. 2006. Perturbations anthropiques du réseau hydrographique du bassin de la Garonne, cas des métaux et des nitrates. These de Doct., Univ. Toulouse III - Paul Sabatier, 476p.

Champaud J. 1983. Villes et Campagnes $d u$ Cameroun de l'Ouest. Edn de l'ORSTOM: Paris, 508p.

Chen WF. 2003. Excitation-Emission Matrix Regional Integration to quantify spectra for a dissolved matter organic matter. Env. Sc. Techn., 37: 5701-5710.

Coble PG. 1996. Characterization of marine and terrestrial dom in seawater using Excitation-Emission Matrix spectroscopy. Marine Chemistry, 51: 325-346.

Colandini V. 1997. Effets des structures réservoirs à revêtement poreux sur les eaux pluviales : qualité des eaux et devenir des métaux lourds. Th. Univ. de Pau et des Pays de l'Adour, 254p.
Cotrim Da Cunha L. 2000. Caractérisation de la matière organique particulaire et dissoute dans un fleuve méditerranéen, le Tech. Thèse Doct. Univ. Perpignan, xxx p.

Devallois V. 2006. Transferts et mobilité des éléments traces métalliques dans la colonne sédimentaire des hydrosystèmes continentaux. Th. Doct. Univ. Provence, $304 p$.

Djeuda Tchapnga HB, Tanawa E, Ngnikam E. 2001. L'Eau au Cameroun. Tome 1: Approvisionnement en Eau Potable. Presses Universitaires de Yaoundé: Yaoundé; 359 p.

Djuikom E, Temgoua E, Jugnia LB, Nola M, Baane M, 2009. Pollution bactériologique des puits d'eau utilisés par les populations dans la Commune Urbaine de Douala Cameroun. Int. J. Biol. Chem. Sci., 3(5): 967-978.

Durand C. 2003. Caractérisation physicochimique des produits de l'assainissement pluvial. Origine et devenir des des métaux traces et des polluants organiques. Th. Doct. Univ. Poitiers, 268p.

Durrieu G. 2004. Etude de la distribution en métaux traces et en matière organique en milieu côtier méditerranéen apportés par une petite rivière. Mém. DEA Chimie et microbiologie de l'eau, Univ. Poitiers, $38 \mathrm{p}$.

Forstner U, Wittmann GTW. 1981. Metal Pollution in the Aquatic Environment. Springer-Verlag: New York; 486p.

Gossart P. 2001. Contribution à l'étude des interactions de la matière organique des sols avec les métaux lourds. Etude structurale et analytique de molécules modèles. Th. Doct. Université des Sciences et Technologies de Lille, 134p.

Hieng IO. 2003. Etude des Paramètres Géotechniques des Sols au Cameroun. Edns CLE: Yaoundé, Cameroun.

INS. 2005. Annuaire Statistique du Cameroun 2004. Institut National de Statistique.

Journal Officiel de l'Union européenne. 2003. Directive 2003/40/CE de la commission du 16 mai 2003, 6p. 
Jourdan B, Piantone P, Lerouge C, Guyonnet D. 2005. Atténuation des métaux à l'aval de sites de stockage de déchets - Synthèse bibliographique. BRGM, $110 \mathrm{p}$.

Ketchemen-Tandia B, Ntamak-Nida MJ, Wonkam C, Ngo Boum S. 2004. Physicochimie de la nappe phréatique des sables quaternaires de Douala (Cameroun). 20 colloque de Géologie africaine - Orléans, France, 2-7 juin 2004.

Koul'a NRT. 2006. contribution du forage de Koumassi dans l'amélioration de l'approvisionnement en eau par la Société Nationale des Eaux du Cameroun à Douala. Mem. Ing. Agron. Univ. Dschang, Cameroun, 74p.

Merian E. 1991. Metals and their Compounds in the Environment. VCH: Weinheim; $1439 \mathrm{p}$.

Ndjama J, Kamgang Kabeyene BV, Sigha Nkamdjou L, Ekodeck G, Tita M Awah 2008. Water supply, sanitation and health risks in Douala, Cameroon. African Journal of Environmental Science and Technology, 2(12): 422-429.

Ngueutchoua G. 1996. Etude des faciès et environnements sédimentaires du quaternaire supérieur du plateau continental camerounais. Th. Doct., Univ. Perpignan, 288p.

Njike Ngaha PR. 1984. Contribution à l'étude géologique, stratigraphique et structurale de la bordure Atlantique du Cameroun. Th. Doct. $3^{\text {ème }}$ cycle, Univ. Yaoundé I, Cameroun, 131p.

OMS (Organisation Mondiale de la Santé). 2006. Directives de Qualité pour l'Eau de Boisson (Vol 1, $3^{\mathrm{e}}$ édn). OMS: Genève.

Regnoult J. 1986. Synthèse Géologique du Cameroun. Ministère des Mines: Yaoundé, Cameroun; 119p.

Serghini A, Fekhaoui M, El Abidi A, Tahri L, Bouissi M, Zaid EH. 2003. Metal contamination of the ground water in Mohammedia (Morocco). Cahiers d'Études et de Recherches Francophones/ Santé, 13(3): 177-182.

Tchameni R. 1997. Géochimie et géochronologie des formations de l'Archéen et du Paléoprotérozoïque du Sud Cameroun (Groupe du Ntem, craton du Congo). Th. Doct., Univ. Orléans, $356 \mathrm{p}$.

Temgoua E, Ngnikam E, Ndongson B. 2009. Drinking water quality: stakes of control and sanitation in the town of Dschang Cameroon. Int. J. Biol. Chem. Sci., 3(3): 441-447.

Zhao H. 2011. Analyse de la matière organique et ses propriétés dans l'environnement naturel en spectroscopie de fluorescence 3D traitée par PARAFAC. Th. Doct., Univ. du Sud Toulon-Var, 164p. 